\title{
Toward tight gamma-ray burst luminosity relations
}

\author{
Shi Qi ${ }^{1,2,3}$ and Tan $\mathrm{Lu}^{1,2,3}$ \\ ${ }^{1}$ Purple Mountain Observatory, Chinese Academy of Sciences, Nanjing 210008, China \\ 2 Joint Center for Particle, Nuclear Physics and Cosmology, Nanjing University - Purple Mountain \\ Observatory, Nanjing 210093, China \\ ${ }^{3}$ Key Laboratory of Dark Matter and Space Astronomy, Chinese Academy of Sciences, Nanjing 210008, \\ China \\ qishi11@gmail.com t.lu@pmo.ac.cn
}

\begin{abstract}
The large scatters of luminosity relations of gamma-ray bursts (GRBs) have been one of the most important reasons that prevent the extensive applications of GRBs in cosmology. Many efforts have been made to seek tight luminosity relations. With the latest sample of 116 GRBs with measured redshift and spectral parameters, we investigate 6 two-dimensional (2D) correlations and 14 derived three-dimensional (3D) correlations of GRBs to explore the possibility of decreasing the intrinsic scatters of the luminosity relations of GRBs. We find the 3D correlation of $E_{\text {peak }}-\tau_{\mathrm{RT}}-L$ to be evidently tighter (at the $2 \sigma$ confidence level) than its corresponding $2 \mathrm{D}$ correlations, i.e., the $E_{\text {peak }}-L$ and $\tau_{\mathrm{RT}}-L$ correlations. In addition, the coefficients before the logarithms of $E_{\text {peak }}$ and $\tau_{\mathrm{RT}}$ in the $E_{\text {peak }}-\tau_{\mathrm{RT}}-L$ correlation are almost exact opposites of each other. Inputting this situation as a prior reduces the relation to $L \propto\left(E_{\text {peak }}^{\prime} / \tau_{\mathrm{RT}}^{\prime}\right)^{0.842 \pm 0.064}$, where $E_{\text {peak }}^{\prime}$ and $\tau_{\mathrm{RT}}^{\prime}$ denote the peak energy and minimum rise time in the GRB rest frame. We discuss how our findings can be interpreted/understood in the framework of the definition of the luminosity (energy released in units of time). Our argument about the connection between the luminosity relations of GRBs and the definition of the luminosity provides a clear direction for exploring tighter luminosity relations of GRBs in the future.
\end{abstract}

Subject headings: gamma-ray burst: general

\section{Introduction}

Gamma-ray bursts (GRBs) are the most luminous astrophysical events observed so far. The high luminosities make them observable at very high redshifts. The measured highest redshift for GRBs has exceeded 8 (e.g., the GRB 090423 with a redshift of $z \approx 8.2$ (Tanvir et al. 2009; Salvaterra et al. 2009) and the GRB 090429B with a photometric redshift of $z \sim 9.4$ (Cucchiara et al. 2011)). Many efforts have been made to apply them to cosmology (see, for example, Dai et al. 2004; Ghirlanda et al. 2004b; Firmani et al. 2005; Lamb et al. 2005; Liang \& Zhang 2005; Xu et al. 2005; Wang \& Dai 2006; Ghirlanda et al. 2006; Schaefer 2007; Wang et al. 2007; Li et al. 2008;
Amati et al. 2008; Basilakos \& Perivolaropoulos 2008; Qi et al. 2008a, b; Kodama et al. 2008; Liang et al. 2008; Wang 2008; Qi et al. 2009; Cardone et al. 2009; Izzo et al. 2009; Liang et al. 2010; Cardone et al. 2010; Wang et al. 2011, etc.). The key for this is to find some luminosity relation that relates the luminosity (e.g., the isotropic peak luminosity $L$ ) or energy (e.g., the isotropic energy $E_{\gamma, \text { iso }}$ or the collimation-corrected energy $E_{\gamma}$ ) of GRBs to their measurable properties (the luminosity indicators). The tighter the relation is, the more accurate and reliable information about the universe we can get from it. Many luminosity relations have been discovered in the literature, e.g., the relations of $\tau_{\text {lag }}-L$ (Norris et al. 2000), $\quad V-L$ (Fenimore \& Ramirez-Ruiz 2000; 
Reichart et al. 2001), $E_{\text {peak }}-E_{\gamma \text {.iso }}$ Amati et al. 2002), $E_{\text {peak }}-E_{\gamma}$ (Ghirlanda et al. 2004a), $E_{\text {peak }}-$ $L$ (Schaefer 2003; Wei \& Gao 2003; Yonetoku et al. 2004), and $\tau_{\mathrm{RT}}-L$ (Schaefer 2007), etc. Here, the spectral lag $\tau_{\text {lag }}$ is the time shift between the hard and soft light curves. The variability $V$ is a quantitative measurement of the spikiness of the light curve, which can be obtained by calculating the normalized variance of the observed light curve around the smoothed light curve. There exist several definitions of $V$, depending mainly on the smoothing time intervals upon which the reference curve is built and the normalization. $E_{\text {peak }}$ is the photon energy at which the $\nu F_{\nu}$ spectrum peaks. The minimum rise time $\tau_{\mathrm{RT}}$ of a GRB light curve is the shortest time over which the light curve rises by half the peak flux of the pulse. In addition, correlations were also found between the transition times of the X-ray light curve from exponential to power law and the X-ray luminosities at the transitions (Dainotti et al. 2008, 2010; Qi \& Lu 2010).

The most crucial problem with GRBs for cosmological application is that their luminosity relations are usually quite scattered, which prevents them from being used as good standard candles as Type Ia supernovae. Therefore, one of the most important directions of cosmological study for GRBs is to seek GRB luminosity relations with sufficiently small intrinsic scatters. Many attempts have been made in literature, among which, a remarkable approach is to explore possible hidden parameters in known correlations in order to reduce the intrinsic scatters. For example, Firmani et al. (2006) claimed that a temporal parameter of the prompt emission, $T_{0.45}$, could reduce the scatter of the correlation of $E_{\text {peak }}-L$ to a negligible value. It was later found that the new proposed relation does not appear to be as tight as it seemed (Rossi et al. 2008; Collazzi \& Schaefer 2008). Tsutsui et al. (2009, 2010) suggested that introducing the parameter $T_{L} \equiv E_{\gamma \text {,iso }} / L$ to the $E_{\text {peak }}-L$ relation could substantially reduce the intrinsic scatters of the correlation of $E_{\text {peak }}-L$ and $E_{\text {peak }}-E_{\gamma \text {,iso }}$ Xu \& Huang (2012) reported that a significantly tighter correlation can be obtained by adding $E_{\gamma \text {,iso }}$ to the correlation discovered in Dainotti et al. (2008, 2010). However, they did not perform the normalization (see the following section of this paper for details about nor- malization) before comparing the intrinsic scatters. Taking into account the normalization, the new relation is in fact looser instead of tighter in the sense of cosmological distance measurement. In $\mathrm{Yu}$ et al. (2009), more general analysis was carried out, investigating 5 two-dimensional (2D) correlations and 10 derived three-dimensional (3D) correlations to explore the possibility of reducing the intrinsic scatters.

Since we have more GRBs at hand now Xiao \& Schaefer 2009; Wang et al. 2011), it is beneficial to update the comparison between $2 \mathrm{D}$ and $3 \mathrm{D}$ luminosity relations of GRBs and to check whether the conclusions drawn from an earlier GRB sample still hold. This is the main aim of this paper. We included more GRB luminosity relations in our analysis than there are in $\mathrm{Yu}$ et al. (2009). Six 2D correlations and 14 derived 3D correlations were investigated. We basically followed the method used in $\mathrm{Yu}$ et al. (2009) for the analysis. To be complete, we briefly describe the method in the following section. The results and discussion are presented in Section 3. A summary is given in the last section.

\section{Methodology}

For the 2D luminosity relations, we consider the following correlations:

$$
\begin{aligned}
\log \frac{L}{1 \mathrm{erg} \mathrm{s}^{-1}} & =a_{1}+b_{1} \log \left[\frac{\tau_{\text {lag }}(1+z)^{-1}}{0.1 \mathrm{~s}}\right], \\
\log \frac{L}{1 \mathrm{erg} \mathrm{s}^{-1}} & =a_{2}+b_{2} \log \left[\frac{V(1+z)}{0.02}\right], \\
\log \frac{L}{1 \mathrm{erg} \mathrm{s}^{-1}} & =a_{3}+b_{3} \log \left[\frac{E_{\mathrm{peak}}(1+z)}{300 \mathrm{keV}}\right], \\
\log \frac{E_{\gamma}}{1 \mathrm{erg}} & =a_{4}+b_{4} \log \left[\frac{E_{\mathrm{peak}}(1+z)}{300 \mathrm{keV}}\right], \\
\log \frac{L}{1 \mathrm{erg} \mathrm{s}-1} & =a_{5}+b_{5} \log \left[\frac{\tau_{\mathrm{RT}}(1+z)^{-1}}{0.1 \mathrm{~s}}\right], \\
\log \frac{E_{\gamma, \text { iso }}}{1 \mathrm{erg}} & =a_{6}+b_{6} \log \left[\frac{E_{\mathrm{peak}}(1+z)}{300 \mathrm{keV}}\right] .
\end{aligned}
$$

The first five of the above correlations are exactly the luminosity relations considered in $\mathrm{Yu}$ et al. (2009). With the latest compilation of GRBs, the $V-L$ relation has become quite scattered (Xiao \& Schaefer 2009; Wang et al. 2011), which makes the parameter $V$ a very poor luminosity indicator. Despite this, we still include this relation in our analysis in 
order to investigate whether $V$ is helpful in reducing the intrinsic scatter when constructing $3 \mathrm{D} \mathrm{lu}-$ minosity relations together with other luminosity indicators. In this paper, we added in our analysis the correlation of Eq. (6), for which more GRBs can be utilized, compared with the similar correlation of Eq. (4), due to its independence from the GRB jet opening angle. In the correlations, the isotropic peak luminosity $L$, the isotropic energy $E_{\gamma, \text { iso }}$, and the collimation-corrected energy $E_{\gamma}$ are derived from the observables of the bolometric peak flux $P_{\text {bolo }}$, the bolometric fluence $S_{\text {bolo }}$, and the beaming factor $F_{\text {beam }}$ through

$$
\begin{aligned}
L & =4 \pi d_{L}^{2} P_{\text {bolo }}, \\
E_{\gamma, \text { iso }} & =4 \pi d_{L}^{2} S_{\text {bolo }}(1+z)^{-1}, \\
E_{\gamma} & =E_{\gamma, \text { iso }} F_{\text {beam }},
\end{aligned}
$$

where $d_{L}$ is the luminosity distance, which depends on the cosmological model and is inversely proportional to the value of the Hubble parameter of today. As in Yu et al. (2009), we adopt the flat $\Lambda$ CDM model with $\Omega_{m}=0.27$ and replace $d_{L}$ with $\bar{d}_{L}=\frac{H_{0}}{c} d_{L} \times 1 \mathrm{~cm}$ in the calculation, so that the dependence on the Hubble constant is absorbed into the intercepts $a_{i}$ of the linear luminosity relations.

The method used to extend the 2D luminosity relations to $3 \mathrm{D}$ ones is also basically the same as that adopted in Yu et al. (2009), except we have more 3D luminosity relations here due to the addition of the correlation of Eq. (6). First, for later convenience when denoting specific correlations, we summarize the equations for the $2 \mathrm{D}$ luminosity relations above as

$$
y^{(i)}=c_{0}^{(i, i)}+c_{1}^{(i, i)} x^{(i)},
$$

where

$$
\begin{gathered}
x^{(1)}=\log \left[\frac{\tau_{\operatorname{lag}}(1+z)^{-1}}{0.1 \mathrm{~s}}\right], \\
x^{(2)}=\log \left[\frac{V(1+z)}{0.02}\right], \\
x^{(3)}=x^{(4)}=x^{(6)}=\log \left[\frac{E_{\mathrm{peak}}(1+z)}{300 \mathrm{keV}}\right], \\
x^{(5)}=\log \left[\frac{\tau_{\mathrm{RT}}(1+z)^{-1}}{0.1 \mathrm{~s}}\right], \\
y^{(1)}=y^{(2)}=y^{(3)}=y^{(5)}=\log \frac{L}{1 \mathrm{erg} \mathrm{s}^{-1}},
\end{gathered}
$$

$$
\begin{aligned}
y^{(4)} & =\log \frac{E_{\gamma}}{1 \mathrm{erg}}, \\
y^{(6)} & =\log \frac{E_{\gamma, \text { iso }}}{1 \mathrm{erg}},
\end{aligned}
$$

and

$$
c_{0}^{(i, i)}=a_{i}, \quad c_{1}^{(i, i)}=b_{i} .
$$

The coefficients $c$ are given two superscripts to incorporate the $3 \mathrm{D}$ correlations introduced below. We denote a luminosity relation by the superscript pair of the corresponding $c$. We let $i<j$ for all the $3 \mathrm{D}$ luminosity relations $(i, j)$ to avoid duplication, and we classify them into the following three groups for convenience:

1. Correlations between the luminosity (the isotropic peak luminosity L) and two luminosity indicators. For $(i, j)$ with both $i$ and $j$ in $(1,2,3,5)$, the 3D luminosity relations are

$$
y^{(i)}=c_{0}^{(i, j)}+c_{1}^{(i, j)} x^{(i)}+c_{2}^{(i, j)} x^{(j)} .
$$

2. Correlations between energy (the isotropic energy $E_{\gamma, \text { iso }}$ or the collimation-corrected energy $\left.E_{\gamma}\right)$ and two luminosity indicators. For $(i, j)=(1,4),(2,4)$, or $(4,5)$, the 3D luminosity relations are

$$
y^{(4)}=c_{0}^{(i, j)}+c_{1}^{(i, j)} x^{(i)}+c_{2}^{(i, j)} x^{(j)},
$$

and for $(i, j)=(1,6),(2,6)$, or $(5,6)$ they are

$$
y^{(6)}=c_{0}^{(i, j)}+c_{1}^{(i, j)} x^{(i)}+c_{2}^{(i, j)} x^{(j)} .
$$

3. Correlations between the luminosity, the energy $E_{\gamma, \text { iso }}$ or $E_{\gamma}$, and the peak energy $E_{\text {peak }}$. For $(i, j)=(3,4)$ or $(3,6)$, the 3D luminosity relations are

$$
y^{(i)}=c_{0}^{(i, j)}+c_{1}^{(i, j)} x^{(i)}+c_{2}^{(i, j)} y^{(j)} .
$$

A luminosity relation can be multiplied by an equal constant on both sides of the equation without actually changing the correlation itself. However, the multiplication would change the intrinsic scatter of the correlation by a factor of the absolute value of the constant multiplied on the equation. To compare the intrinsic scatters of the luminosity relations, we divide both sides of 
Eq. (22) by a factor of $1-c_{2}^{(i, j)}$, so that $\log \left(d_{L}\right)$ terms have the same coefficient in all the luminosity relations discussed here. We normalize the luminosity relations in this way because, in addition to being helpful in understanding the GRBs themselves, the luminosity relations are mainly aimed at distance measurements.

To explore possible hidden parameters in the 2D luminosity relations, we compare the intrinsic scatters of $3 \mathrm{D}$ luminosity relations with those of corresponding 2D ones. The principle is that the two luminosity relations compared with each other should have two parameters in common, so that we can conclude whether the intrinsic scatter is reduced by introducing the third parameter. Following this principle, for the 3D luminosity relations $(i, j)$ in the first and third classes, i.e., those of Eq. (19) and (22), they are compared with 2D luminosity relations $(i, i)$ and $(j, j)$, and for the 3D luminosity relations in the second class, i.e., those of Eq. (20) and (21), they are compared with 2 D luminosity relations $(4,4)$ and $(6,6)$ respectively. Only when the intrinsic scatter of a $3 \mathrm{D}$ luminosity relation is smaller than that of all its corresponding 2D luminosity relation(s) can we say that the intrinsic scatter is reduced.

In the fit of the luminosity relations, we used the techniques presented in D'Agostini (2005). Using the correlation of Eq. (19) as an example, the joint likelihood function for the coefficients $c$ and the intrinsic scatter $\sigma_{\text {int }}$ is given as

$$
\begin{aligned}
\mathcal{L}\left(c, \sigma_{\mathrm{int}}\right) & \propto \prod_{k} \frac{1}{\sqrt{\sigma_{\mathrm{int}}^{2}+\sigma_{y_{k}^{(i)}}^{2}+c_{1}^{2} \sigma_{x_{k}^{(i)}}^{2}+c_{2}^{2} \sigma_{x_{k}^{(j)}}^{2}}} \\
& \times \exp \left[-\frac{\left(y_{k}^{(i)}-c_{0}-c_{1} x_{k}^{(i)}-c_{2} x_{k}^{(j)}\right)^{2}}{2\left(\sigma_{\mathrm{int}}^{2}+\sigma_{y_{k}^{(i)}}^{2}+c_{1}^{2} \sigma_{x_{k}^{(i)}}^{2}+c_{2}^{2} \sigma_{x_{k}^{(j)}}^{2}\right)}\right],
\end{aligned}
$$

where $k$ runs over GRBs with corresponding quantities available. The joint likelihood functions are similar for other 3D luminosity relations. For the 2D luminosity relations, the joint likelihood functions can be obtained by setting $c_{2}=0$.

For the GRB data, we used the compilation in Wang et al. (2011), which includes 116 GRBs. For this compilation, the data of the luminosity indicators are taken from Xiao \& Schaefer (2009). The size of this GRB sample is almost twice as large as the compilation in Schaefer (2007), which was used in the previous study to compare 2D and 3D luminosity relations ( $\mathrm{Yu}$ et al. 2009). In addition, significant improvements have been made in the calculation of the luminosity indicators for the updated compilation. When considering error propagation from a quantity, say $\xi$ with error $\sigma_{\xi}$, to its $\log$ arithm, we set $\left[\log \left(\xi+\sigma_{\xi}^{+}\right)+\log \left(\xi-\sigma_{\xi}^{-}\right)\right] / 2$ and $\left[\log \left(\xi+\sigma_{\xi}^{+}\right)-\log \left(\xi-\sigma_{\xi}^{-}\right)\right] / 2$ as the center value and the error of the logarithm correspondingly. This requires $\xi>\sigma_{\xi}^{-}$(the quantities we are interested in here are all positive). Due to the limitation of the data, for a given luminosity relation $(i, j)$, not all the GRBs have all of the needed observational quantities available and satisfy $\xi>\sigma_{\xi}^{-}$ at the same time. By set $(i, j)$ we denote the maximum GRB set that can be used in the luminosity relation $(i, j)$. The numbers of GRBs of different sets are presented in Table 1

\section{Results and discussion}

We summarize our results for the fits to the luminosity relations and comparisons between $2 \mathrm{D}$ and 3D correlations in Table 1. We list our findings from the table as follows:

1. For the correlations common with those studied in Yu et al. (2009), including both $2 \mathrm{D}$ and $3 \mathrm{D}$ ones, almost all the intrinsic scatters have increased with the updated GRB sample. For the $2 \mathrm{D}$ ones, only the $\tau_{\mathrm{RT}}-L$ relation retains a comparable intrinsic scatter.

2. For the cases of $(1,3)$ and $(1,5)$, the intrinsic scatters of the 3D correlations are reduced at the $1 \sigma$ confidence level compared with their corresponding $2 \mathrm{D}$ correlations, and for the case of $(3,5)$, the intrinsic scatter is reduced at the $2 \sigma$ confidence level. No other statistically significant reduction of the intrinsic scatters is found.

3 . For the case of $(3,5)$, the values of the coefficients $c_{1}$ and $c_{2}$ are almost exact opposites of each other. For the case of $(1,3)$, the values of $c_{1}$ and $c_{2}$ are also rough opposites of each other.

Item 1 reminds us once again of the complexity of GRBs. Correlations should be tested against a large enough sample to determine how good they 


\begin{tabular}{|c|c|c|c|c|c|c|}
\hline \multirow[t]{3}{*}{$(i, j)$} & 1 & 2 & 3 & 4 & 5 & 6 \\
\hline & $\tau_{\text {lag }}$ & V & $E_{\text {peak }}$ & $E_{\text {peak }}$ & $\tau_{\mathrm{RT}}$ & $E_{\text {peak }}$ \\
\hline & $L$ & $L$ & $L$ & $E_{\gamma}$ & $L$ & $E_{\gamma, \text { iso }}$ \\
\hline \multirow{5}{*}{$\begin{array}{l}1 \\
\tau_{\text {lag }}\end{array}$} & 53 & 46 & 53 & 13 & 49 & 46 \\
\hline & $\left(-3.958_{-0.067}^{+0.067},-0.7\right.$ & $1.04_{-0.13}^{+0.13},-0.70_{-0.14}^{+0.14}, 0.14_{-0}^{+0}$ & $\left(-4.014_{-0.059}^{+0.059},-0.57_{-0.10}^{+0.10}, 0.67_{-0.16}^{+0.17}\right.$ & $\left(-5.565_{-0.094}^{+0.093},-0.06_{-0.16}^{+0.16}, 1.33_{-0.25}^{+0.24}\right)$ & $\left(-3.750_{-0.079}^{+0.081},-0.41_{-0.12}^{+0.12},-0.71_{-0.17}^{+0.17}\right)$ & $\left(-3.471_{-0.074}^{+0.074},-0.43_{-0.12}^{+0.12}, 0.88_{-0.19}^{+0.19}\right)$ \\
\hline & $0.476_{-0.046}^{+0.054}$ & $0.479_{-0.050}^{+0.060}$ & $0.402_{-0.041}^{+0.048}$ & $0.25_{-0.08}^{+0.10}$ & $0.404_{-0.041}^{+0.049}$ & $0.452_{-0.049}^{+0.059}$ \\
\hline & & {$\left[-0.002_{-0.075}^{+0.073}, 0.192_{-0.080}^{+0.078}\right]$} & {$\left[0.074_{-0.066}^{+0.068}, 0.135_{-0.061}^{+0.059}\right]$} & {$\left[0.05_{-0.12}^{+0.11}\right]$} & {$\left[0.072_{-0.067}^{+0.068}, 0.069_{-0.064}^{+0.064}\right]$} & {$\left[0.037_{-0.069}^{+0.065}\right]$} \\
\hline & & $L$ & $L$ & $E_{\gamma}$ & $L$ & $E_{\gamma, \text { iso }}$ \\
\hline \multirow{4}{*}{$\begin{array}{l}2 \\
V\end{array}$} & & 81 & 81 & 21 & 64 & 68 \\
\hline & & $\left(-4.39_{-0.14}^{+0.14}, 0.60_{-0.15}^{+0.15}\right)$ & $\left(-4.31_{-0.11}^{+0.11}, 0.33_{-0.13}^{+0.13}, 1.13_{-0.15}^{+0.15}\right)$ & $\left(-5.71_{-0.14}^{+0.14}, 0.14_{-0.14}^{+0.14}, 1.45_{-0.16}^{+0.16}\right)$ & $\left(-3.68_{-0.14}^{+0.14}, 0.20_{-0.13}^{+0.13},-1.03_{-0.15}^{+0.15}\right)$ & $\left(-3.62_{-0.13}^{+0.13}, 0.26_{-0.13}^{+0.13}, 1.10_{-0.16}^{+0.16}\right)$ \\
\hline & & $0.672_{-0.053}^{+0.060}$ & $0.503_{-0.041}^{+0.046}$ & $0.207_{-0.055}^{+0.067}$ & $0.428_{-0.040}^{+0.046}$ & $0.483_{-0.042}^{+0.049}$ \\
\hline & & & {$\left[0.168_{-0.070}^{+0.073}, 0.034_{-0.060}^{+0.059}\right]$} & {$\left[0.096_{-0.096}^{+0.099}\right.$} & {$\left[0.244_{-0.070}^{+0.072}, 0.045_{-0.062}^{+0.062}\right]$} & {$\left[0.007_{-0.061}^{+0.060}\right]$} \\
\hline \multirow{5}{*}{$\begin{array}{l}3 \\
E_{\text {peak }}\end{array}$} & & & ${ }_{L}$ & $L, E_{\gamma}$ & $L$ & $L, E_{\gamma, \text { iso }}$ \\
\hline & & & 116 & 24 & 72 & 101 \\
\hline & & & $\left(-4.134_{-0.053}^{+0.053}, 1.40_{-0.12}^{+0.12}\right)$ & $\left(-1.3_{-1.3}^{+1.3}, 0.49_{-0.38}^{+0.38}, 0.46_{-0.24}^{+0.23}\right)$ & $\left(-3.739_{-0.062}^{+0.061}, 0.84_{-0.12}^{+0.12},-0.85_{-0.11}^{+0.11}\right)$ & $\left(-1.24_{-0.27}^{+0.27}, 0.15_{-0.14}^{+0.14}, 0.827_{-0.075}^{+0.074}\right)$ \\
\hline & & & $0.538_{-0.038}^{+0.042}$ & $0.78_{-0.25}^{+0.60}$ & $0.348_{-0.033}^{+0.038}$ & $2.2_{-0.7}^{+1.6}$ \\
\hline & & & & {$\left[-0.24_{-0.60}^{+0.25},-0.48_{-0.60}^{+0.26}\right]$} & {$\left[0.189_{-0.053}^{+0.054}, 0.125_{-0.056}^{+0.058}\right]$} & {$\left[-1.7_{-1.6}^{+0.7},-1.8_{-1.6}^{+0.7}\right]$} \\
\hline \multirow{5}{*}{$\begin{array}{l}4 \\
E_{\text {peak }}\end{array}$} & & & & $E_{\gamma}$ & $E_{\gamma}$ & \\
\hline & $\mathrm{cr}$ & & & 24 & 22 & \\
\hline & & $\cdots$ & $\cdots$ & $\left(-5.639_{-0.074}^{+0.071}, 1.47_{-0.20}^{+0.19}\right)$ & $\left(-5.60_{-0.11}^{+0.11}, 1.40_{-0.25}^{+0.24},-0.12_{-0.23}^{+0.22}\right)$ & \\
\hline & & & & $0.304_{-0.068}^{+0.082}$ & $0.342_{-0.074}^{+0.092}$ & \\
\hline & & & & & {$\left[-0.04_{-0.11}^{+0.11}\right]$} & \\
\hline \multirow{5}{*}{$\begin{array}{l}5 \\
\tau_{\mathrm{RT}}\end{array}$} & & & & & $L$ & $E_{\gamma, \text { iso }}$ \\
\hline & & & & & 72 & 62 \\
\hline & & $\cdots$ & $\cdots$ & & $\left(-3.563_{-0.074}^{+0.074},-1.12_{-0.14}^{+0.14}\right)$ & $\left(-3.316_{-0.082}^{+0.081},-0.32_{-0.15}^{+0.15}, 0.99_{-0.17}^{+0.16}\right)$ \\
\hline & & & & & $0.473_{-0.042}^{+0.048}$ & $0.469_{-0.043}^{+0.050}$ \\
\hline & & & & & & {$\left[0.020_{-0.063}^{+0.061}\right]$} \\
\hline \multirow{4}{*}{$\begin{array}{l}6 \\
E_{\text {peak }}\end{array}$} & & & & & & $E_{\gamma, \text { iso }}$ \\
\hline & & & & & & 101 \\
\hline & & & & & & $\left(-3.532_{-0.053}^{+0.052}, 1.47_{-0.12}^{+0.12}\right)$ \\
\hline & & & & & & $0.490_{-0.038}^{+0.042}$ \\
\hline
\end{tabular}

Table 1: Fit of 2D and 3D luminosity relations. The first row and the first column of the table are the indices denoting the luminosity relations and the corresponding luminosity indicators. In every cell of the table, the first row is the luminosity and/or energy involved in the relations which, together with the luminosity indicators, tell what the correlations are about. The second row of every cell is the number of GRBs of set $(i, j)$, the vector below enclosed by parentheses is the vector of $c$ for the luminosity relation $(i, j)$, and what follows next is the intrinsic scatter. For 3D luminosity relations, the reduction of the intrinsic scatters compared with corresponding 2D luminosity relations is presented in the brackets. The statistics in the table are for the median values and the errors with the $1 \sigma(68.3 \%)$ confidence level. 
are. The intrinsic scatter derived from a small sample may be significantly affected by the selection effect of the sample itself. Item 2 is basically consistent with the previous studies of $\mathrm{Yu}$ et al. (2009), in which the intrinsic scatters are reduced at $1 \sigma$ and $2 \sigma$ confidence levels, respectively, for the cases of $(1,3)$ and $(3,5)$, i.e., the $\tau_{\text {lag }}-E_{\text {peak }}{ }^{-}$ $L$ and $E_{\text {peak }}-\tau_{\mathrm{RT}}-L$ correlations. With the new GRB sample adopted here, the intrinsic scatter is also reduced at the $1 \sigma$ confidence level for the case of $(1,5)$, i.e., the $\tau_{\mathrm{lag}}-\tau_{\mathrm{RT}}-L$ correlation, the edges of the two $1 \sigma$ confidence intervals of the magnitude of reduction of the intrinsic scatter for $(1,5)$ are both very close to zero, which reduces its reliability. The correlation $(3,5)$ turns out to be the only $3 \mathrm{D}$ correlation that appears very robust in the test of the reduction of the intrinsic scatter.

The correlation $(3,5)$ is among $E_{\text {peak }}, \tau_{\mathrm{RT}}$, and $L$. It is a relation among an energy scale, a timescale, and the luminosity. This naturally makes us think of the definition of the luminosity (energy released in units of time). As can be seen from the large scatters of the 2D luminosity relations, it is most likely that, due to their complexity, the luminosities of GRBs cannot be well determined from only one quantity. However, according to the definition of the luminosity, we should in principle be able to calculate the luminosity from a characteristic energy scale and a characteristic timescale of GRBs. If we could find two measurable quantities (e.g., an energy scale and a timescale) that are strongly correlated with the assumed characteristic energy scale and timescale, then we would find a 3D luminosity relation between the luminosity and the two measurable quantities. The quality of the relation obviously depends on the strength of the correlations between the two quantities and the assumed characteristic energy scale and timescale, and is also related to the degree of match between the two quantities in the sense of the match between energy scale and timescale in the definition of the luminosity (i.e., the energy scale and timescale chosen to calculate the luminosity should correspond to each other). The intrinsic scatter of such a 3D luminosity relation is expected to be significantly reduced compared with its corresponding $2 \mathrm{D}$ ones. The situation described in item 2 suggests $E_{\text {peak }}$ and $\tau_{\mathrm{RT}}$ as one such pair of measurable quantities.

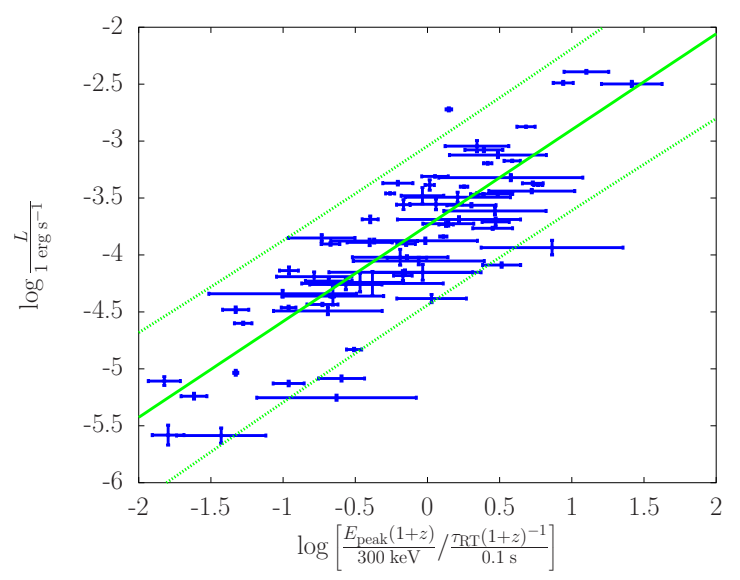

Fig. 1.- Best fit of the GRBs to the luminosity relation of Eq. (24) and the corresponding $2 \sigma$ confidence region. In the calculation of the $L$, the dependence of the luminosity distance on the Hubble constant is absorbed into the intercept of the relation.

What makes the correlation $(3,5)$ more interesting is the situation described in item 3 . Following the discussion above, the almost exact opposition of $c_{1}$ and $c_{2}$ suggests a considerably high degree of match between $E_{\text {peak }}$ and $\tau_{\mathrm{RT}}$ considering that, in the definition of the luminosity, the indices for the energy scale and the timescale are opposite. In comparison, the degree of match between $E_{\text {peak }}$ and $\tau_{\text {lag }}$ is slightly weaker. The situation of the almost exact opposite for the correlation $(3,5)$ does not show up in the results of Yu et al. (2009). However, remember that, for the GRB data used here, not only is the sample size much larger, but significant improvements also have been made in the calculation of the luminosity indicators, so we have reasons to treat the situation as more than just a coincidence. If we input the opposition of $c_{1}$ and $c_{2}$ as a prior for the correlation, then the relation is reduced to a $2 \mathrm{D}$ one, that is,

$\log \frac{L}{1 \mathrm{erg} \mathrm{s}^{-1}}=a+b \log \left[\frac{E_{\mathrm{peak}}(1+z)}{300 \mathrm{keV}} / \frac{\tau_{\mathrm{RT}}(1+z)^{-1}}{0.1 \mathrm{~s}}\right]$.

A fit to this relation gives the result of $a=$ $-3.742_{-0.048}^{+0.047}, b=0.842_{-0.064}^{+0.064}$, and the intrinsic scatter $\sigma_{\text {int }}=0.346_{-0.032}^{+0.037}$. We present the relation in Figure 1 Using $E_{\text {peak }}^{\prime}$ and $\tau_{\mathrm{RT}}^{\prime}$ to denote the peak energy and minimum rise time in the GRB 
rest frame, the relation can be expressed as

$$
L \propto\left(\frac{E_{\text {peak }}^{\prime}}{\tau_{\mathrm{RT}}^{\prime}}\right)^{0.842 \pm 0.064}
$$

Following the definition of the luminosity, if appropriate luminosity is used on the left-hand side of Eq. (24) or Eq. (25), the coefficient $b$ in Eq. (24) or the index in Eq. (25) should be 1. However, in practice, there are various definitions of luminosities, e.g., peak or averaged luminosity and isotropic or collimation-corrected luminosity. It is unclear which luminosity is attached to the pair of energy scale and timescale used on the right-hand side of equations, i.e., $E_{\text {peak }}$ and $\tau_{\text {RT }}$. What we adopt in the relations is the isotropic peak luminosity $L$. Thus, the coefficient $b$ in Eq. (24) or the index in Eq. (25) may be interpreted as the correction factor between the luminosity attached to the pair of $E_{\text {peak }}$ and $\tau_{\mathrm{RT}}$ and the isotropic peak luminosity.

For a clear and complete physical interpretation of the $E_{\text {peak }}-\tau_{\mathrm{RT}}-L$ correlation, it should be explained how $E_{\text {peak }}$ and $\tau_{\text {RT }}$ are correlated with the assumed energy scale and timescale. This is an issue that needs further detailed study, which is out of the scope of this short paper. Here, we present some general speculation on this. One may note that $E_{\text {peak }}$ is not a radiated energy, but the photon energy at which the $\nu F_{\nu}$ spectrum peaks. The calculation of the luminosity needs the radiated energy. $E_{\text {peak }}$ may relate itself with a radiated energy through a physical process that releases photons with the frequency at which $\nu F_{\nu}$ spectrum peaks, or it may just be a dominant parameter in the spectrum that can be used as a representation of the bolometric energy released by the GRB, as can be seen from $E_{\text {peak }}-E_{\gamma \text {,iso }}$ and $E_{\text {peak }}-$ $E_{\gamma}$ correlations $\left(E_{\gamma, \text { iso }}\right.$ and $E_{\gamma}$ cannot replace the role of $E_{\text {peak }}$ here, mostly because their calculation depends on the luminosity distance and the intrinsic scatters are enlarged in the procedure of normalization, while $E_{\text {peak }}$ is directly measured from the observation and is independent of the cosmological model). We think that $\tau_{\mathrm{RT}}$ stands out in our investigation most likely because $\tau_{\mathrm{RT}}$ is the minimum rise time. Though requiring it to be minimum sounds like a very simple operation in the data processing, it could be a very important step for extracting the clean physics from the complex GRBs, thus eliminating intrinsic scatters from many irrelevant physical processes.

We would also like to mention the $3 \mathrm{D}$ correlation of $(3,6)$, i.e., the $E_{\text {peak }}-E_{\gamma \text {,iso }}-L$ correlation and the $E_{p}-T_{L}-L_{p}$ correlation referenced in Tsutsui et al. (2009, 2010). Using the notation adopted here, the latter is given by

$$
\log L=A+B \log \left[E_{\text {peak }}(1+z)\right]+C \log T_{L},
$$

where $T_{L}=E_{\gamma, \text { iso }} / L$. Substituting $T_{L}$ into the equation, the correlation can be rewritten as

$$
(1+C) \log L=A+B \log \left[E_{\text {peak }}(1+z)\right]+C \log E_{\gamma, \text { iso }} .
$$

Comparing it with Eq. (22), we can see that this relation is in fact the same as the $3 \mathrm{D}$ luminosity relation $(3,6)$, and it has been already normalized. However, the conclusion we draw here for the correlation conflicts with that in Tsutsui et al. (2009, 2010). Tsutsui et al. (2009, 2010) claimed that the intrinsic scatter of the correlation $E_{p}-T_{L}-$ $L_{p}$ is smaller than that of both the $E_{\text {peak }}-L$ and $E_{\text {peak }}-E_{\gamma, \text { iso }}$ correlations. Our result shows that the intrinsic scatter of the correlation of $(3,6)$ is not reduced compared with its corresponding $2 \mathrm{D}$ correlations. In fact, its intrinsic scatter even appears a little larger. The advantages of our analysis here are that we used more GRBs, which helped us reduce the selection effect from the sample itself, and that we adopted a sophisticated statistical method, in which not only the intrinsic scatter but also its error can be derived, so that we can judge the statistical significance of the reduction of the intrinsic scatters.

\section{Summary}

As a further step on in seeking tight luminosity relations of GRBs, we fitted the latest data of GRBs with measured redshift and spectral parameters to $62 \mathrm{D}$ correlations and 14 derived 3D correlations and compared their intrinsic scatters to explore possible hidden parameters in the $2 \mathrm{D}$ correlations. Compared with the analysis of an early sample of GRBs, the intrinsic scatters of most of the luminosity relations have increased, which reminds us of the complexity of GRBs. Correlations should be tested against a large enough sample to reduce the possible selection effects from the sample itself. Our result shows that the $E_{\text {peak }}-\tau_{\mathrm{RT}}-L$ 
correlation appears to be significantly tighter (at the $2 \sigma$ confidence level) than its corresponding $2 \mathrm{D}$ correlations, i.e., the $E_{\text {peak }}-L$ and $\tau_{\mathrm{RT}}-L$ correlations. What is more interesting is that, in the $E_{\text {peak }}-\tau_{\mathrm{RT}}-L$ correlation, the coefficients before the logarithms of $E_{\text {peak }}$ and $\tau_{\mathrm{RT}}$ are almost exact opposites of each other. If this situation is input as a prior, the correlation is reduced to a $2 \mathrm{D}$ one, $L \propto\left(E_{\text {peak }}^{\prime} / \tau_{\mathrm{RT}}^{\prime}\right)^{0.842 \pm 0.064}$, where $E_{\text {peak }}^{\prime}$ and $\tau_{\mathrm{RT}}^{\prime}$ denote the peak energy and minimum rise time in the GRB rest frame. We interpret/understand the result in the framework of the definition of the luminosity (energy released in units of time). Due to the complexity of GRBs, it is unlikely that we can accurately determine their luminosity through only one quantity, as can be seen from the large scatters of the 2D luminosity relations. And in principle, we should be able to calculate the luminosity from a characteristic energy scale and a characteristic timescale of GRBs. Our result suggests that $E_{\text {peak }}$ and $\tau_{\mathrm{RT}}$ are a pair of measurable quantities which are sufficiently correlated with the assumed characteristic energy scale and timescale and that there is a high degree of match between them (in the sense of the match between energy scale and timescale in the definition of the luminosity), so we may construct a better luminosity relation with them. Since there are different definitions of the luminosity, the index of 0.842 in $L \propto\left(E_{\text {peak }}^{\prime} / \tau_{\mathrm{RT}}^{\prime}\right)^{0.842 \pm 0.064}$ may be interpreted as the correction factor between the luminosity attached to the pair of $E_{\text {peak }}$ and $\tau_{\mathrm{RT}}$ and the isotropic peak luminosity $L$.

Our argument about the connection between the luminosity relations of GRBs and the definition of the luminosity provides a clear direction for exploring tighter luminosity relations of GRBs in the future. It should also be easier to seek correlations between measurable quantities and the assumed characteristic energy scale and timescale related to the luminosity than to directly seek the relations between the measurable quantities and the luminosity. Our findings about the $E_{\text {peak }}{ }^{-}$ $\tau_{\mathrm{RT}}-L$ correlation illustrate this approach.

We thank the anonymous referee for helpful comments and suggestions. This work was supported by the National Natural Science Foundation of China under grant no. 10973039, the Chinese Academy of Sciences under grant no. KJCX2-
EW-W01, and the China Postdoctoral Science Foundation under grant no. 20100471421 (for Shi Qi).

\section{REFERENCES}

Amati, L., et al. 2002, Astron. Astrophys., 390, 81

—. 2008, Mon. Not. Roy. Astron. Soc., 391, 577

Basilakos, S., \& Perivolaropoulos, L. 2008, Mon. Not. Roy. Astron. Soc., 391, 411

Cardone, V., Capozziello, S., \& Dainotti, M. 2009, Mon. Not. Roy. Astron. Soc., 400, 775

Cardone, V., Dainotti, M., Capozziello, S., \& Willingale, R. 2010, Mon. Not. Roy. Astron. Soc., 408,1181

Collazzi, A. C., \& Schaefer, B. E. 2008, Astrophys. J., 688,456

Cucchiara, A., Levan, A., Fox, D., Tanvir, N., Ukwatta, T., et al. 2011, Astrophys.J., 736, 7

D'Agostini, G. 2005, arXiv:physics/0511182

Dai, Z. G., Liang, E. W., \& Xu, D. 2004, Astrophys. J., 612, L101

Dainotti, M. G., Cardone, V. F., \& Capozziello, S. 2008, MNRAS, 391, L79

Dainotti, M. G., Willingale, R., Capozziello, S., Cardone, V. F., \& Ostrowski, M. 2010, Astrophys. J., 722, L215

Fenimore, E. E., \& Ramirez-Ruiz, E. 2000, arXiv:astro-ph/0004176

Firmani, C., Ghisellini, G., Avila-Reese, V., \& Ghirlanda, G. 2006, Mon. Not. Roy. Astron. Soc., 370,185

Firmani, C., Ghisellini, G., Ghirlanda, G., \& Avila-Reese, V. 2005, Mon. Not. Roy. Astron. Soc., 360, L1

Ghirlanda, G., Ghisellini, G., \& Firmani, C. 2006, New J. Phys., 8, 123

Ghirlanda, G., Ghisellini, G., \& Lazzati, D. 2004a, Astrophys. J., 616, 331

Ghirlanda, G., Ghisellini, G., Lazzati, D., \& Firmani, C. 2004b, Astrophys. J., 613, L13 
Izzo, L., Capozziello, S., Covone, G., \& Capaccioli, M. 2009, Astron. Astrophys., 508, 63

Kodama, Y., et al. 2008, MNRAS, 391, L1

Lamb, D. Q., Ricker, G., Lazzati, D., Ghirlanda, G., Ghisellini, G., et al. 2005, arXiv:astro-ph/0507362

Li, H., et al. 2008, Astrophys. J., 680, 92

Liang, E.-W., \& Zhang, B. 2005, Astrophys. J., 633, 611

Liang, N., Wu, P., \& Zhang, S. N. 2010, Phys.Rev., D81, 083518

Liang, N., Xiao, W. K., Liu, Y., \& Zhang, S. N. 2008, Astrophys. J., 685, 354

Norris, J. P., Marani, G. F., \& Bonnell, J. T. 2000, Astrophys. J., 534, 248

Qi, S., \& Lu, T. 2010, Astrophys. J., 717, 1274

Qi, S., Lu, T., \& Wang, F.-Y. 2009, Mon. Not. Roy. Astron. Soc., 398, L78

Qi, S., Wang, F.-Y., \& Lu, T. 2008a, Astron. Astrophys., 483, 49

-. 2008b, Astron. Astrophys., 487, 853

Reichart, D. E., et al. 2001, Astrophys. J., 552, 57

Rossi, F., et al. 2008, Mon. Not. Roy. Astron. Soc., 388, 1284

Salvaterra, R., et al. 2009, Nature, 461, 1258

Schaefer, B. E. 2003, Astrophys. J., 583, L71

—. 2007, Astrophys. J., 660, 16

Tanvir, N. R., et al. 2009, Nature, 461, 1254

Tsutsui, R., Nakamura, T., Yonetoku, D., Murakami, T., \& Takahashi, K. 2010, arXiv:1012.3009

Tsutsui, R., et al. 2009, JCAP, 0908, 015

Wang, F., Qi, S., \& Dai, Z. 2011, Mon. Not. Roy. Astron. Soc., 415, 3423

Wang, F. Y., \& Dai, Z.-G. 2006, Mon. Not. Roy. Astron. Soc., 368, 371
Wang, F. Y., Dai, Z. G., \& Zhu, Z.-H. 2007, Astrophys. J., 667, 1

Wang, Y. 2008, Phys. Rev., D78, 123532

Wei, D. M., \& Gao, W. H. 2003, Mon. Not. Roy. Astron. Soc., 345, 743

Xiao, L., \& Schaefer, B. E. 2009, Astrophys. J., 707,387

Xu, D., Dai, Z., \& Liang, E. W. 2005, Astrophys. J., 633, 603

Xu, M., \& Huang, Y. 2012, Astron. Astrophys., 538, A134

Yonetoku, D., et al. 2004, Astrophys. J., 609, 935

Yu, B., Qi, S., \& Lu, T. 2009, Astrophys. J., 705, L15

This 2-column preprint was prepared with the AAS LATEX macros v5.2. 